\title{
$X X X$. The rate of recombination of ions in gases under different pressures
}

\section{R.K. McClung M.A.}

To cite this article: R.K. McClung M.A. (1902) XXX. The rate of recombination of ions in gases under different pressures, Philosophical Magazine Series 6, 3:15, 283-305, DOI: 10.1080/14786440209462765

To link to this article: http://dx.doi.org/10.1080/14786440209462765

册 Published online: 08 Jun 2010.

Submit your article to this journal $[\pi$

Џll Article views: 4

Q View related articles $\square$ 
centimetre of the substance. This, for a crystal of the cubic class, is, in Voigt's mathematical theory, the analogue to the electric effect discovered in quartz by the brothers Curie, and measured by aid of thin metal foils attached to the two faces of the plate and metallically connected to the two principal electrodes of an electrometer.

XXX. The Rate of Recombination of Ions in Gases under Different Pressures. By R. K. McClung, M.A., formerly Demonstrator of Physics, Mc Gill University, Montreal ; 1851 Exhibition Scholar, MeGill University, Trinity Collere, Cambridge*.

A NY gas which has been exposed to Röntgen rays, retains A the ionization thus produced for a short time after the source of radiation has been removed. The negative and positive ions take an appreciable time to recombine with one another; and it is necessary in some cases to take into consideration the rate at which these ions disappear by recombination. This question of the rate of recombination of ions has been investigated by Prof. Rutherford (Phil. Mag. Nov. 1897) for air and some other gases at atmospheric pressure. In that paper he points out that if, when the rate of production becomes equal to the rate of recombination, the rays be then stopped, the rate at which the conducting particles decay is given by the equation

$$
\frac{d n}{d t}=-a n^{2}, . \quad . \quad . \quad . \quad . \quad . .
$$

where $n$ is the number of ions per c.c. in the gas at any time $t$ after the rays have ceased, and $\alpha$ is a constant for any given gas at ordinary atmospheric pressure. If $\mathrm{N}$ be the maximum number of ions per c.c. when a steady state has been reached before the cessation of the rays, then the above equation becomes by integration

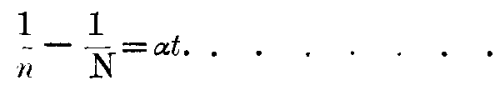

Olject of Investigation.

At the suggestion of Professor Rutherford, the present research was undertaken in order to ascertain the conditions which exist in regard to the recombination of the ions in gases under pressures other than atmospheric pressure, and to find the relation between the rates of recombination at different pressures. The objects of the present investigation have been therefore :-

(1) To determine whether the law of recombination, as set * Communicated by Prof. E. Rutherford. 
forth in equation (1), holds true for air under pressures ahove and below an atmosphere.

(2) If it does hold true, to find the relation which the quantity $\alpha$ at any given pressure bears to that at any other pressure.

(3) To make an absolute determination of $a$.

This quantity a may be called the coefficient of recombination.

For this purpose the method employed by Professor Rutherford in his previous investigation was adopted in principle, but with an apparatus differing somewhat in detail.

In order to investigate the law, as given by equation (2), it is necessary to be able to measure the maximum number of ions in a given volume of the gas when a steady state has been reached, and also to measure the number remaining at the end of any given interval of time after the rays have ceased. For this purpose, then, the following arrangement of apparatus was adopted.

\section{Description of Apparatus.}

The complete arrangement of the several parts and connexions is shown in the diagram (fig. 1).

Fig. 1.

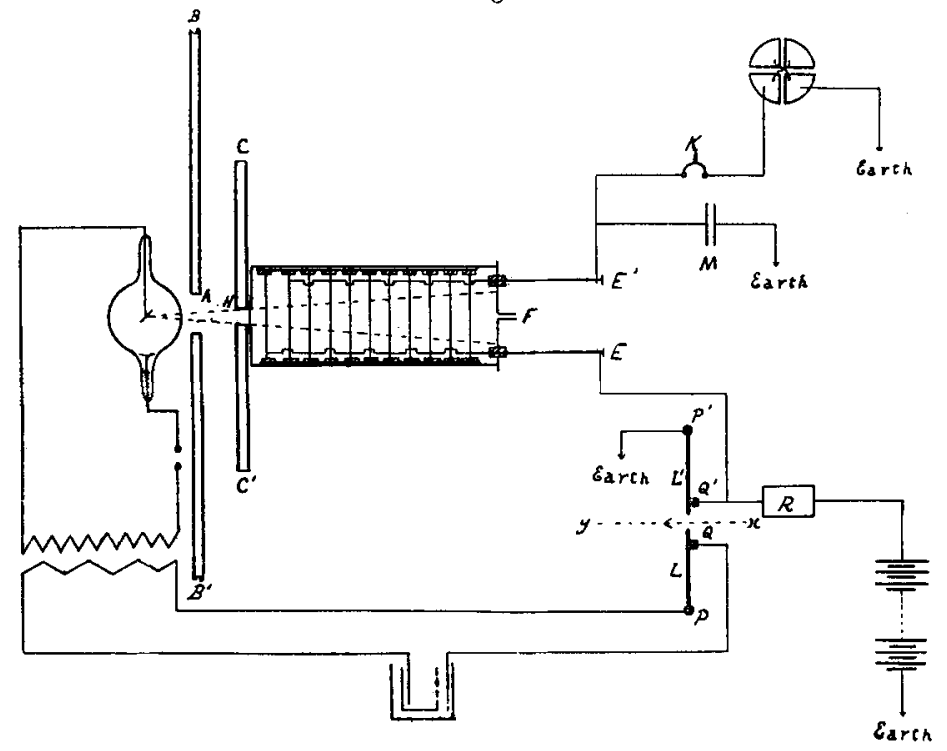

The bulb used to produce the Röntgen rays was of the usnal form of automatic focus-tube, with an alternative 
spark-gap connexion, and was driven by a large Ruhmkorff induction-coil. The coil was run by the current from the 110-volt circuit, with a Wehnelt interrupter of slow frequency in series. In order to intensify the discharge in the focustube, a spark-gap of about a centimetre and a half was placed directly in series with the secondary of the coil and the electrodes of the tube.

The induction-coil and $x$-ray bulb were completely inclosed in a small metal room connected to earth and the rays issued through a small orifice $A$ in a thick lead plate $B B^{\prime}$. A short distance in front of this was placed another thick lead plate $\mathrm{CC}^{\prime}$. The rays passed through a small rectangular orifice $\mathrm{H}$ in this plate and then into a brass cylinder through an aluminium window. This cylinder, which is shown more in detail in fig. 2, contained the electrodes between which the leak was measured.

Fig. 2,

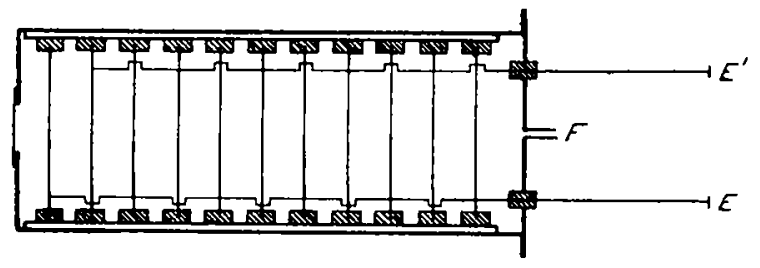

The electrodes employed consisted of extremely thin aluninium-foil. stretched on narrow zinc rings, the rings being for the purpose of support. 'These rings were supported on ebonite blocks, which were rigidly fastened to a metal frame, which could easily be removed from the cylinder. These thin plates were eleven in number, and were placed at distances of about two centimetres apart. Six of them, namely the two end ones and the four alternate ones of the remainder, were all connected together and also to the terminal $\mathrm{E}$, while the other five alternate plates were connected to $\mathrm{E}^{\prime}$.

This arrangement of electrodes was adopted in order to secure a large volume of gas to be ionized, and thus obtain a larger effect than could be obtained otherwise, while at the same time the electrodes were close enough together to secure a saturation-current with a reasonable voltage. All the ions could thus reach the electrodes in a very short time. This precaution was essential as it was necessary that all the ions should reach the electrodes before disappearing by recombination. An arrangement consisting of a guard- 
ring was tried, but the effect produced was so small that it was not suitable for measurement, and so was abandoned.

Thin aluminium-foil was used in the first place because, being so very thin, it would not absorb the rays to any appreciable extent, and, secondly, because the secondary radiation produced at the surface of metals is small in the case of aluminium. The actual thickness of the foil used was only 00036 of a centimetre. Before finally adopting the aluminium a set of electrodes consisting of paper covered with powdered graphite and then soaked with dilute acid so. as to make them conductors, was tried. These gave very satisfactory results while they lasted; but they were not durable, and so had to be abandoned also. The final arrangement of the aluminium plates seemed to be the most satisfactory, and was therefore adopted. The terminals connected to the electrodes passed through ebonite corks in the end of the cylinder to secure insulation:

The cylinder was made so that the air might be exhausted from it through the opening $\mathrm{F}$, and it was also made strong enough to stand considerable internal pressure.

In order to measure the maximum number of ions in a given volume of the gas when a steady state had been reached, and also the number remaining at the end of any given interval of time, it was necessary to be able to apply the electromotive force to the electrode $\mathrm{E}$ at the same instant that. the rays ceased, and also at any given time after the cessation of the rays. For this purpose the following method was used. $L$ and $I{ }^{\prime}$ were two contact-breakers, pivoted at $P$ and $\mathrm{P}^{\prime}$ and making contact with $\mathrm{Q}$ and $\mathrm{Q}^{\prime}$ respectively. The primary circuit of the induction-coil was closed through the contact-maker L. The lever $L^{\prime}$ was connected to earth, while $\mathrm{Q}^{\prime}$ was connected to the electrode $\mathrm{E}$ and also, through a very large resistance $R$, to one pole of a battery of small accumulators, the other pole of the battery being connected to earth. The resistance $R$ which was used was a carbon one of about a megohm.

Now since the resistance between $\mathrm{Q}^{\prime}$ and $\mathrm{E}$ was extremely small compared with $R$, then, if the lever $L^{\prime}$ were closed against $Q^{\prime}$, the plates connected with $\mathrm{E}$ would be practically at zero potential, since $L^{\prime}$ is connected to earth. But as soon as the contact was broken between $L^{\prime}$ and $Q^{\prime}$, then $E$ would be instantly charged up to the potential of the battery. The contacts at $\mathrm{Q}$ and $\mathrm{Q}^{\prime}$ were broken by means of a heavy pendulum which swung along the path $x y$. If the levers were placed directly opposite to each other, the rays could be stopped and the electromotive force applied to $E$ at the same 
instant; while if they were separated from each other along the path $x y$, the rays could be stopped and the E.M.F. applied at a known interval afterward. This interval could be varied by varying the distance between the levers $\mathrm{L}$ and $L^{\prime}$.

The time taken by the pendulum to pass from one contactbreaker to the other was determined by calibrating the pendulum. This was done by attacbing a smoked-glass plate to the pendulum and obtaining a trace on this plate made by a vibrating tuning-fork of known frequency. By this means the time taken by the pendulum to pass from one point to another could be calculated. The electrode $\mathrm{E}^{\prime}$ was connected, through a suitable key at $K$, to one pair of quadrants of the electrometer, while the other pair was connected to earth.

\section{Method of Observation.}

The following was the method of observation for determining the values of $\mathrm{N}$ and $n$ in equation (2). The inductioncoil circuit was closed and the rays allowed to pass into the cylinder for about ten seconds, to insure the ionization reaching a maximum. This point was tested by running the hulb for a longer time before applying the E.M.F., and it was found that about ten seconds was quite sufficient for the jonization to reach a maximum. The pendulum was then released, which stopped the rays and applied the E.M.F. to $\mathrm{E}$ by breaking the contacts. During all this time both pairs of quadrants of the electrometer were to earth. In order to avoid the violent throw of the elecirometer-needle, which would oceur by induction if the pair of quadrants connected to $E^{\prime}$ were insulated before the E.M.F. was applied to E, the connexion between the electrometer and $\mathrm{E}^{\prime}$ was broken by the key $K$ until after the lever $L^{\prime}$ was replaced against $Q^{\prime}$, which was done immediately after the passage of the pendulum. After replacing $L^{\prime}$, the charge in the plates connected with $\mathrm{E}^{\prime}$, due to the ions which passed across on the application of the E.M.F. to $\mathrm{E}$, was thrown into the electrometer by closing the contact $\mathrm{K}$. The deflexion of the electrometer-needle should then be proportional to the number of ions in the gas between the electrodes at the instant of the application of the E.M.F. to E, if the E.M.F. is large enough to produce saturation. The potential-gradient employed in most of the observations was about 110 volts per centimetre.

One of the great difficulties encountered in this investigation was the want of constancy in the intensity of the radiation given out by the bulb. To obviate this to some extent when 
taking a series of observations, the bulb was run at regular intervals as far as possible. Besides that, instead of depending on a single observation in any one case, several observations were made, and then the mean of these taken. All through the investigation this system of averages has been adopted.

\section{Theoretical Considerations.}

Before proceeding to the actual results obtained by experiment, some theoretical questions must be considered.

Suppose that the number of ions per c.c. which are being produced at any given point in the gas per second is $q$, and suppose $n$ equals the actual number per c.c. in the gas at the same point, then it has been shown (J. J. Thomson and E. Rutherford, Phil. Mag. Nov. 1896) that when a steady state has been reached, that is, when the rate of production is equal to the rate of recombination,

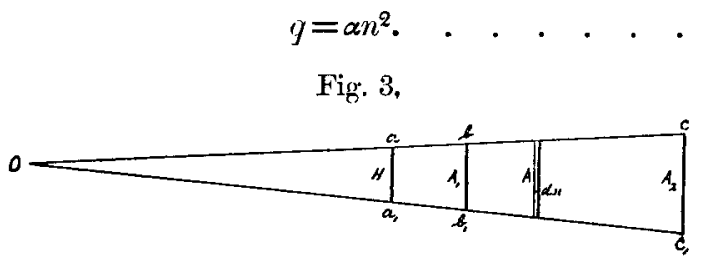

In fig. 3 let $\mathrm{O}$ represent the source of the rays, and let $\mathrm{OCC}_{1}$ represent the cone of rays passing through the rectangular orifice $\mathrm{H}$ (fig. 1) and thence into the cylinder. Let $b b_{1} c_{1} c$ represent the part of the cone included between the two extreme plates within the cylinder. Suppose $\mathrm{H}, \mathrm{A}_{1}$, and $\mathrm{A}_{2}$ to represent the cross-sectional areas of the cone at the orifice and the two plates, respectively. Let $l$ and $d$ represent the distances of $a a_{1}$ and $b b_{1}$ respectively from $O$, while $l$ is the distance between the plates $b b_{1}$ and $c c_{1}$.

Let $q_{0}=$ the number of ions per c.c. produced per second at the plate $b b_{1}$.

Let $Q=$ total number produced per second in the volume between $b b_{1}$ and $c c_{1}$;

$$
\therefore \quad \mathrm{Q}=\int q d \mathrm{~V},
$$

where $q=$ the number of ions per c.c. at a distance $x$ from $O$, and $d \mathrm{~V}=\mathrm{A} d x$, the cross-sectional area at the distance $x$ being $A$.

$$
\mathrm{A}=\frac{x^{2}}{d^{2}} \mathrm{~A}_{1}
$$

and since the number of ions per c.c. varies inversely as the 
square of the distance from the source, neglecting the absorption of the rays by the air,

$$
q=\frac{d^{2}}{x^{2}} q_{0}
$$

This absorption of the air may be neglected without causing any serious error; for it has been found ( $E$. Rntherford and R. K. McClung, Phil. Trans. Roy. Soc. vol. cxcvi. 1901) that only about 3 per cent. of strong radiation was absorbed in passing through a metre of air at atmospheric pressure. The absorption was shown, by the same authors, to be proportional to the pressure of the gas. In the present case, the distance between the two plates $b b_{1}$ and $c c_{1}$ was only 20 centimetres; so the absorption would be very small, and therefore negligible.

Therefore, supplying these values in the above equation, we get

$$
\begin{aligned}
\mathrm{Q} & =\int_{d}^{d+l} \frac{d^{2}}{x^{2}} q_{0} \cdot \frac{x^{2}}{d^{2}} \mathrm{~A}_{1} d x \\
& =q_{0} \mathrm{~A}_{1} l . . .
\end{aligned}
$$

Again, let $\mathrm{N}=$ total number of ions in the same volume at the cessation of the rays;

$$
\therefore \mathrm{N}=\int n d \mathrm{~V},
$$

where $n=$ residual number per c.c. at the distance $x$ from $O$.

$$
\begin{aligned}
\therefore \quad \mathrm{N} & =\int \sqrt{\frac{\bar{q}}{\alpha}} d \mathrm{~V}, \text { since } q=\alpha n^{2} \\
& =\frac{1}{\sqrt{\alpha}} \int_{d}^{d+l} \sqrt{\frac{d^{2}}{x^{2}} q_{0}} \cdot \frac{x^{2}}{d^{2}} \mathrm{~A}_{1} d x \\
& =l \mathrm{~A}_{1} \sqrt{\frac{\bar{q}_{0}}{\alpha}} \times\left(1+\frac{l}{2 d}\right) ; . \\
\therefore \quad \alpha & =l^{2} \mathrm{~A}_{1}^{2}\left(1+\frac{l}{2 d}\right)^{2} \frac{g_{0}}{\mathrm{~N}^{2}} .
\end{aligned}
$$

But $q_{0}=\frac{\mathrm{Q}}{\mathrm{A}_{1} l}$ from equation (4);

$$
\therefore \quad \alpha=l \mathrm{~A}_{1}\left(1+\frac{l}{2 d}\right)^{2} \frac{\mathrm{Q}}{\mathrm{N}^{2}} . \cdots . .
$$

This equation gives the value of the constant $\alpha$ in absolute measure, since the quantities $A_{1}, l$, and $d$ can be measured

Phil. Mag. S. 6. Vol. 3. No. 15. March 1902. U 
and $\mathrm{Q}$ and $\mathrm{N}$ determined. This equation may be written

$$
a=\mathrm{K} \frac{\mathrm{Q}}{\mathrm{N}^{2}}, \quad \cdot \quad \cdot \quad \cdot \quad \cdot \quad \cdot
$$

where $\mathrm{K}$ is equal to the constant $l \mathrm{~A}_{1}\left(1+\frac{l}{2 d}\right)^{2}$, which depends entirely upon the dimensions and position of the apparatus.

The value obtained for $a$ will be discussed later in the paper.

The theory of recombination may be considered also in regard to the time taken by the maximum number of ions to fall to half their value.

Let $n_{0}=$ maximum number of ions per c.c. at a point distant $x$ from $\mathrm{O}$ (fig. 3).

Let $n_{t}=$ number per c.c. at the end of a time $t$ after the rays have ceased.

Then by equation (2)

$$
\frac{1}{n_{t}}-\frac{1}{n_{0}}=\alpha t .
$$

The decrease in time $t$ per c.c. $=n_{0}-n_{t}$.

The decrease in a thin cross-sectional area $A$, of a thickness $d x$, would be $\mathrm{A}\left(n_{0}-n_{t}\right) d x$.

$$
\begin{aligned}
& \frac{1}{n_{t}}=\frac{1}{n_{0}}+\alpha t ; \\
\therefore \quad & n_{0}-n_{t}=\frac{n_{0}^{2} \alpha t}{1+n_{0} \alpha t} . \\
& \alpha n_{0}^{2}=q=\frac{q_{0} d^{2}}{x^{2}} ; \\
\therefore \quad & n_{0}{ }^{2}=\frac{q_{0}}{\alpha} \cdot \frac{d^{2}}{x^{2}} .
\end{aligned}
$$

Also

Therefore the decrease in the cross-sectional area $A$ in time $t$ is equal to

$$
\begin{aligned}
& \frac{\mathrm{A}_{1} x^{2}}{d^{2}}\left(\frac{q_{0} d^{2}}{\alpha_{2} x^{2}} \cdot \frac{\alpha t}{1+a t \frac{d}{x} \sqrt{\frac{q_{0}}{\alpha}}}\right) d x \\
& \quad=\frac{\mathrm{A}_{1} q_{0} t x}{x+t d \sqrt{q_{0}^{\alpha}}} d x \\
& \quad=\mathrm{A}_{1} q_{0} t\left(1-\frac{t d \sqrt{\frac{q_{0} \alpha}{}}}{x+t d \sqrt{q_{0^{\alpha}}}}\right) d x ;
\end{aligned}
$$


Therefore the total decrease in the number of ions in the volume $b b_{1} c_{1} c$ in the time $t$

$$
\begin{aligned}
& =\int_{d}^{d+l} \mathrm{~A}_{1} q_{0} t\left(1-\frac{t d \sqrt{q_{0}^{\alpha}}}{x+t d \sqrt{q_{0}^{\alpha}}}\right) d x \\
& =A_{1} g_{0} t\left\{l-t d \sqrt{q_{0} \alpha} \log _{\epsilon}\left(\frac{d+l+t d \sqrt{q_{0} \alpha}}{d+t d \sqrt{q_{0} \alpha}}\right)\right\} .
\end{aligned}
$$

If we let $\mathrm{T}$ be the time that it takes the number of ions to fall to half their value, then the total decrease in the time $T$

$$
=\mathrm{A}_{1} q_{0} \mathrm{~T}\left\{l-\mathrm{T} d \sqrt{g_{0} \alpha} \log _{\epsilon}\left(\frac{d+l+\mathrm{T} d \sqrt{q_{0} \alpha}}{d+\mathrm{T} d \sqrt{q_{0} \alpha}}\right)\right\} .
$$

But the maximum number of ions is equal to

$$
\begin{aligned}
& l \mathrm{~A}_{1}\left(1+\frac{l}{2 d}\right) \sqrt{\frac{q_{0}}{\alpha}} \text { by equation (4); } \\
& \therefore \quad \mathrm{A}_{1} g_{0} \mathrm{~T}\left\{l-\mathrm{T} d \sqrt{q_{0} \alpha} \log _{e}\left(\frac{d+l+\mathrm{T} d}{d+\mathrm{T} d} \overline{\sqrt{q_{0} \alpha}} \overline{q_{0}^{\alpha}}\right)\right\} \\
& =\frac{1}{2} l \mathrm{~A}_{1}\left(1+\frac{l}{2 \bar{d}}\right) \sqrt{\frac{\overrightarrow{q_{0}}}{\alpha}} .
\end{aligned}
$$

This equation for obtaining the value of $T$ is incapable of an exact solution. An approximate solution may, however, be obtained as follows :-

$$
\mathrm{A}_{1} q_{0} \mathrm{~T}\left\{l-\mathrm{B} \log _{e}\left(\frac{d+l+\mathrm{B}}{d+\mathrm{B}}\right)\right\}=\frac{1}{2} l \mathrm{~A}_{1}\left(1+\frac{l}{2 d}\right) \sqrt{\frac{q_{0}}{\alpha}},
$$

where $\mathrm{B}=\mathrm{T} d \sqrt{q_{0} \alpha}$,

$$
\therefore \mathrm{A}_{1} q_{0} \mathrm{~T}\left\{l-\mathrm{B} \log _{\mathrm{c}}\left(1+\frac{l}{d+\mathrm{B}}\right)\right\}=\frac{1}{2} l \mathrm{~A}_{1}\left(1+\frac{l}{2 d}\right) \sqrt{\frac{q_{0}}{a}} \text {; }
$$

$\therefore$ by expanding $\log _{e}\left(1+\frac{l}{d+\mathrm{B}}\right)$,

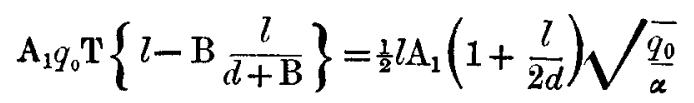

as a first approximation, provided that $l$ is small compared with $d+\mathrm{B}$.

It is found by supplying the experimental values of the quantities involved in the expression $\frac{l}{d+\mathrm{B}}$, that the second term in the expansion of the logarithm amounts to approxi- 
mately $\cdot 125$; and therefore the above expression is a fair value for the first approximation.

$$
\begin{aligned}
& \therefore \quad \mathrm{A}_{1} q_{0} \mathrm{~T} l\left(\frac{d}{\mathrm{~B}+d}\right)=\frac{1}{2} l \mathrm{~A}_{1}\left(1+\frac{l}{2 d}\right) \sqrt{\frac{\overline{q_{0}}}{\alpha}} ; \\
& \therefore \quad \mathrm{T}=\frac{1}{2} \cdot \frac{1}{\sqrt{q_{0} \alpha}}\left(1+\frac{l}{2 d}\right) \frac{\mathrm{B}+d}{d} ; \\
& =\frac{1}{2 \sqrt{q_{0} a}}\left(1+\frac{l}{2 d}\right) \frac{\mathrm{T} d \sqrt{q_{0} \alpha}+d}{d} \\
& =\frac{1}{2}\left(1+\frac{l}{2 d}\right)\left(\mathrm{T}+\frac{1}{\sqrt{q_{0} \alpha}}\right) \text {; } \\
& =\frac{2 d+l}{4 d \sqrt{q_{0} \alpha}} \frac{4 d}{2 d-l} ; \\
& =\frac{1}{\sqrt{q_{0} \alpha}} \cdot \frac{2 d+l}{2 d-l} \\
& =\frac{2 d}{2 d+l} \cdot \frac{\mathrm{N}}{\overline{\mathrm{Q}}} \cdot \frac{2 d+l}{2 d-l} \text { by equations (4) and (6); } \\
& =\frac{2 d}{2 d-l} \cdot \frac{\mathrm{N}}{\mathrm{Q}} \cdot \cdot \cdot \cdot \cdot \cdot \cdot \cdot \cdot .
\end{aligned}
$$

This gives a fairly close approximation for the time taken for the number of ions in the given volume in the cylinder to fall to half of their maximum value.

Having discussed the mathematical theory, we are now in a position to give some experimental results in support of the above theory.

Experimental Proof of the Law of Recombination.

We have endeavoured to establish the foregoing theory at the various pressures by obtaining experimental curves showing the relation between the number of ions and the corresponding time-interval after the cessation of the rays and then comparing them with the theoretical values, as deduced from equation (2). Curves were obtained for different pressures, varying from three atmospheres to 125 of an atmosphere. A considerable number of such experimental curves were obtained, some of which are shown in figs. $4,5,6$, and 7 . III

In these eurves, the ordinates represent deflexions, which are proportional to $n$, while the abscissæ represent the corresponding times in seconds. The experimental points in each curve are marked with a cross, while the theoretical points 
deduced from equation (2) are marked with a circle. Only the experimental curve is drawn in each case in order to avoid confusion by having the two curves so close together. The theoretical points are obtained by choosing two experimental

Fig 4.

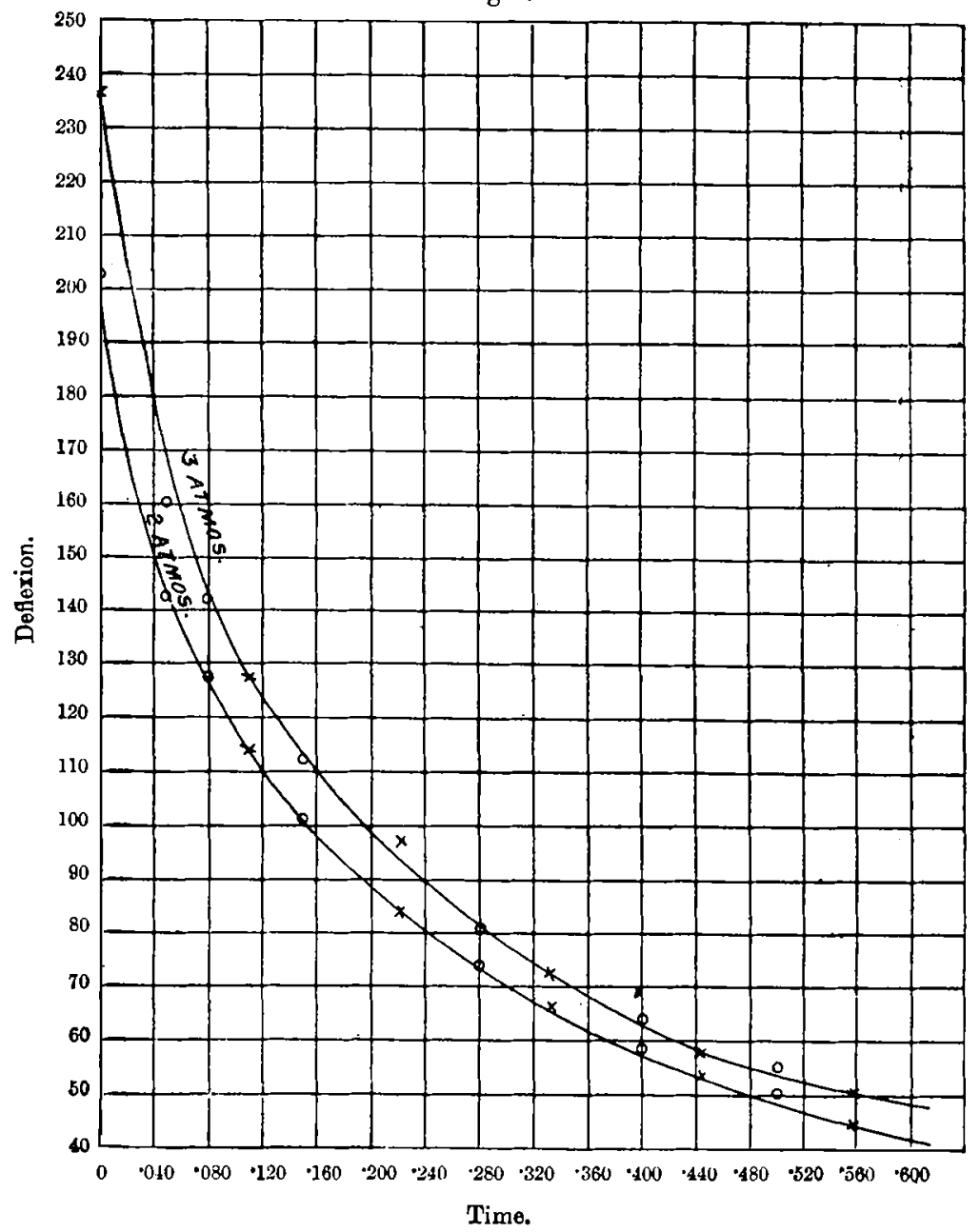

points on the curve with which to determine $a$ and $N$, and having obtained these, then the different values of $n$ are calculated from the formula for arbitrarily chosen values of $t$. On referring to the diagrams it is seen that the experimental 
and theoretical curves correspond very closely with each other. Any slight disagreement is well within the limits of experimental error, especially taking into consideration the fact that the intensity of the rays may vary slightly during the course of taking the observations necessary for one such curve.

Fig. 5.

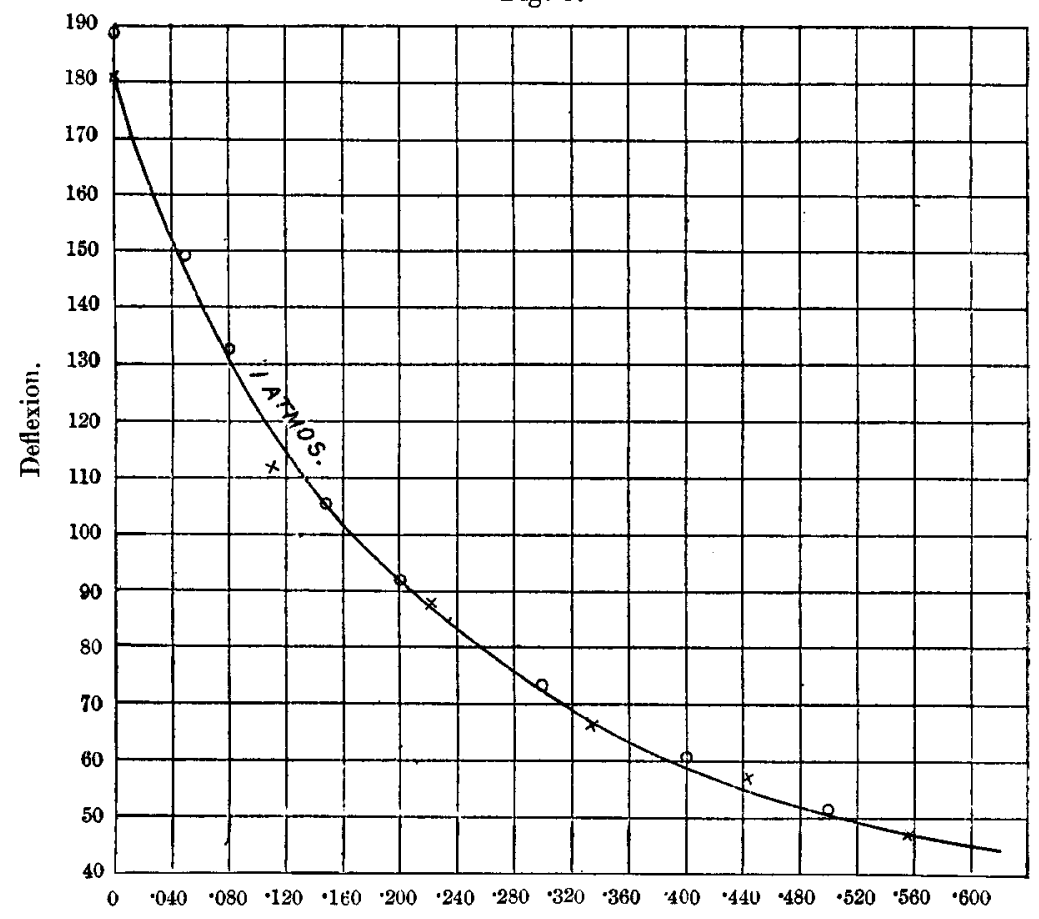

Time.

The agreement is quite close enough to show the accuracy of the theory. The experimental investigation therefore supports the theory, and we see that the same law of recombination holds true at different pressures, at least for the range of pressures investigated.

It was not easy to obtain curves for pressures much below 125 of an atmosphere, for at the very low pressures the effects obtained by this method were so small that they could not be relied upon with any great degree of certainty. However, there are no indications that the law does not hold below this pressure. 
Ions in Gases under Different Pressures.

Fig. 6.

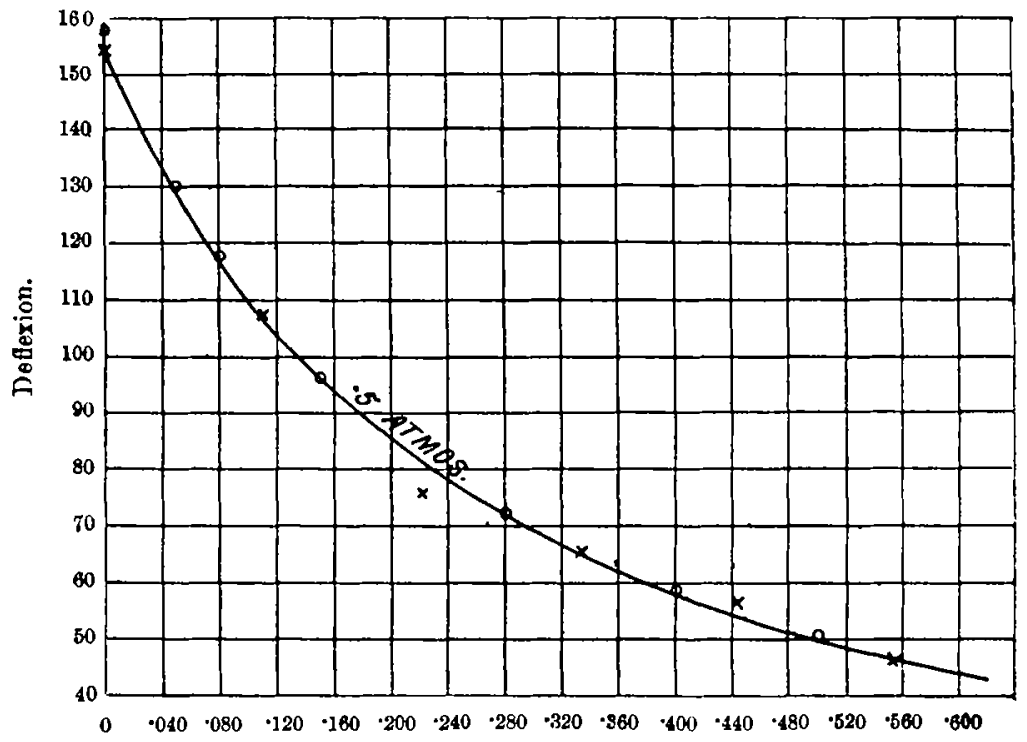

Tine.

Fig. 7.

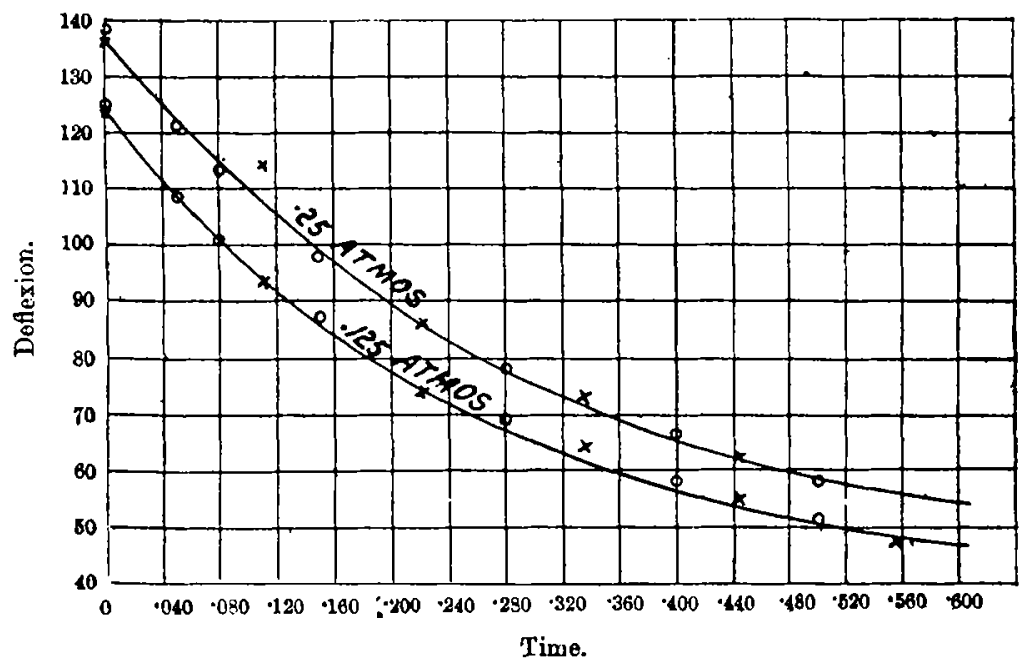


From these curves we see, what we should naturally expect, that the rate at which the ions recombine is much greater at the high pressures than at the low ones, owing, of course, to the fact that at the high pressures there are many more ions produced in a given volume of the gas, and therefore the chance of their coming in contact with one another is much greater than at low pressures.

If we consider the theory from the point of view of the time taken for the maximum number of ions to fall to half their value, we get a further confirmation of it from experiment. By determining the ratio of $\mathrm{N}$ to $\mathrm{Q}$ at each pressure and substituting these values, along with the values of $d$ and $l$ in centimetres in equation (8), we obtain the value of $T$ in seconds in each case. Comparing these calculated values with the values of $\mathrm{T}$ obtained directly from the curves, we find the agreement fairly close, considering the fact that formula (8) is only an approximate formula, and also that a slight variation in the intensity of the rays would cause a corresponding deviation in the shape of the curve, and therefore a slight alteration in the value of $T$ obtained from it. A comparison of these values of $T$ is given for several different pressures in Table I.

\section{TABLF $I$.}

\begin{tabular}{|c|c|c|c|c|c|}
\hline Pressure. & $\begin{array}{l}\text { Deflexion } \\
\text { for } N .\end{array}$ & $\begin{array}{l}\text { J)eflexion } \\
\text { for } Q \text {. }\end{array}$ & $\begin{array}{l}\text { Value of } \\
\frac{2 d}{2 d-l}\end{array}$ & $\begin{array}{l}\text { Calculated } \\
\text { value of } T \text {. }\end{array}$ & $\begin{array}{l}\text { Value of } \mathrm{T} \\
\text { obtained } \\
\text { from curve. }\end{array}$ \\
\hline 3 atmos. & 236 & 143 & $1 \cdot 66$ & $\cdot 114$ & $\cdot 137$ \\
\hline$"$ & 196 & 112 & $1 \cdot 66$ & $\cdot 122$ & $\cdot 161$ \\
\hline $1 \quad$ & 181 & 51 & $1 \cdot 66$ & $\cdot 247$ & $\cdot 203$ \\
\hline$\frac{1}{2} \quad "$ & 154 & 41 & $1 \cdot 66$ & $\cdot 259$ & $\cdot 249$ \\
\hline " & 136 & 26 & $1 \cdot 66$ & $\cdot 360$ & $\cdot 368$ \\
\hline
\end{tabular}

The agreement between theory and experiment is close enough to show that the theory is quite valid no matter from which point of view it may be considered.

We see, therefore, that the relation $\frac{d n}{d t}=-a n^{2}$ gives actually the rate at which the ions recombine in air, not only at atmospheric pressure, lnat also for air at pressures other than atmospheric, at leasi between the limits already indicated. 
Although it has not been practicable to take observations beyond these limits, yet we think the same law would, in all probability, hold true at other pressures as well.

\section{The Relation of a to Pressure.}

The next question to be considered is the relation which the value of the quantity $\alpha$, at any given pressure, bears to that at any other pressure : in other words, to decide whether the coefficient of recombination is a constant for all pressures, or whether it varies when the pressure varies.

In order to get over the difficulty of any variation in the intensity of the radiation which might occur in the course of taking observations at different pressures, the following method was adopted :-

Selecting two given pressures at a time, sets of observations were taken alternately for the two pressures, and then taking the mean value of the observations for one pressure, I compared them with the mean for the other. By this means it was possible to compare the value of $a$ at various pressures with that at atmospheric pressure.

Suppose $\alpha_{1}$ is the value of the coefficient for air at atmospheric pressure, and $\alpha_{2}$ that any other given pressure, then

$$
\alpha_{1}=\mathrm{K} \frac{\mathrm{Q}_{1}}{\mathrm{~N}_{1}{ }^{2}} \text { and } a_{2}=\mathrm{K}_{\mathrm{N}_{2}{ }^{2}}^{\mathrm{Q}_{2}}
$$

where $Q_{1}, N_{1}$, and $Q_{2}, N_{2}$ are the corresponding ralues of $Q$ and $\mathrm{N}$;

$$
\therefore \frac{\alpha_{1}}{\alpha_{2}}=\frac{\mathrm{Q}_{1}}{\mathrm{Q}_{2}} \cdot \frac{\mathrm{N}_{2}{ }^{2}}{\mathrm{~N}_{1}{ }^{2}} \text {. }
$$

By comparing the values of $\alpha$ by this formula, it was found that the coefficient is not only a constant for any one given pressure, but has the same value at different pressures. In other words, the coefficient of recombination is independent of the pressure. The value of $a$ at several pressures, as compared with the value at atmospheric pressure, is given in Table II. (p. 298).

In the table, $\alpha_{1}$ is the value of $\alpha$ at atmospheric pressure, while $\alpha_{2}$ is the value at the other pressure compared with it.

The numbers given in the last column of Table II. do not show an absolute constancy in the value of $\alpha$ at the different pressures, but the difference from unity, in each case, is well within the limits of experimental error, considering the various sources of error which may come into the results. Since $N$ is squared, any small error which might occur would be intensified by squaring. 
TABLE II.

\begin{tabular}{|c|c|c|c|}
\hline Pressure. & $\begin{array}{l}\text { Deflexion } \\
\text { for } \mathbf{N} \text {. }\end{array}$ & $\begin{array}{l}\text { Deflexion } \\
\text { for } Q \text {. }\end{array}$ & $\frac{\alpha_{1}}{\alpha_{2}}$ \\
\hline $\begin{array}{l}1 \text { atmos. } \\
3 \text { " }\end{array}$ & $\begin{array}{l}121.0 \\
193.0\end{array}$ & $\begin{array}{l}34.4 \\
797\end{array}$ & 1.094 \\
\hline $\begin{array}{ll}1 & \prime \prime \\
2 \cdot 5 & \prime \prime\end{array}$ & $\begin{array}{r}67 \cdot 9 \\
103.2\end{array}$ & $\begin{array}{l}16 \% 5 \\
355\end{array}$ & 1.083 \\
\hline $\begin{array}{l}1 \\
2 \quad ",\end{array}$ & $\begin{array}{l}70 \cdot 4 \\
96 \cdot 0\end{array}$ & $\begin{array}{l}15 \cdot 2 \\
26 \cdot 5\end{array}$ & 1.058 \\
\hline $\begin{array}{ll}1 & \\
1.5 & \prime \prime\end{array}$ & $\begin{array}{l}128 \cdot 6 \\
148.7\end{array}$ & $\begin{array}{l}38 \cdot 2 \\
49 \cdot 4\end{array}$ & 1.028 \\
\hline $\begin{array}{ll}1 & \\
\cdot 5 & "\end{array}$ & $\begin{array}{r}1100 \\
93.5\end{array}$ & $\begin{array}{l}29 \cdot 3 \\
207\end{array}$ & 1.018 \\
\hline${ }^{1} \cdot 25$, & $\begin{array}{l}70 \cdot 0 \\
43.5\end{array}$ & $\begin{array}{r}23.5 \\
9.0\end{array}$ & $1 \cdot 011$ \\
\hline${ }^{1} \cdot 125, "$, & $\begin{array}{l}\mathbf{6 1 . 9} \\
29.9\end{array}$ & $\begin{array}{r}22 \cdot 7 \\
5 \cdot 1\end{array}$ & $1 \cdot 029$ \\
\hline
\end{tabular}

The set of comparisons above were not all made at the same time, but several of the observations were made on different. days, extending over a period of about two weeks, and the comparison in each case was made with air at atmospheric pressure, so they do not represent a single set of values.

We have thus arrived at the fact that not only does the same law of recombination of the ions in air hold at various pressures, but the value of the coefficient of recombination is independent of the pressure of the air in which the ionization takes place. If the value of $\alpha$ is therefore determined for air at atmospheric pressure, it is determined for air at other pressures as well.

\section{Determination of the Absolute Value of $\alpha$.}

We now proceed to determine the absolute value for this coefficient of recombination. Since the value of $\alpha$ is the same for different pressures, it is only necessary to determine it for a single given pressure, so atmospheric pressure has been 
selected for the determination given below. A considerable number of absolute determinations at other pressures have been made as well, which it is not necessary to reproduce here.

On referring to equation (7), it will be seen that it is necessary to make an absolute determination of the three quantities $\mathrm{K}, \mathrm{Q}$, and $\mathrm{N}$, in order to determine $\alpha . \mathrm{K}$ is a constant depending entirely on the dimensions and position of the apparatus. $Q$ is the total number of ions produced per second within the volume of the cone of rays included between the two extreme electrodes, and is proportional to the deflexion of the electrometer-needle per second. $\mathrm{N}$ is the total number of ions in the same volume at the instant that the rays cease, and is proportional to the steady deflexion of the electrometer-needle.

From formula (6)

$$
\mathrm{K}=l \mathrm{~A}_{1}\left(1+\begin{array}{c}
l \\
2 d
\end{array}\right)^{2}
$$

where the quantities $l, \mathrm{~A}_{1}$, and $d$ represent the dimensions as described in fig. 3.

$$
\begin{aligned}
l & =20 \mathrm{cms} . \\
d & =25 \cdot 07 \mathrm{cms} . \\
\mathrm{A}_{1} & =\frac{d^{2}}{h^{2}} \times \mathrm{H},
\end{aligned}
$$

where $\mathrm{H}=$ area of the orifice in the lead plate through which the rays passed before entering the cylinder, and $h$ its distance from the source.

$$
\begin{aligned}
\therefore \quad \mathrm{A}_{1} & =\frac{25 \cdot 07^{2}}{19 \cdot 07^{2}} \times 7 \cdot 14 \\
& =12 \cdot 28 \mathrm{sq} . \mathrm{cms} . \\
\therefore \quad \mathrm{K} & =20 \times 12 \cdot 28\left(1+\frac{20}{50 \cdot 14}\right)^{2} \\
& =478.9 .
\end{aligned}
$$

If $d_{1}=$ deflexion of the electrometer-needle per second due to the passage of the ions to the electrodes ;

$\mathrm{S}=$ deflexion due to a standard Clark cell; and

$c_{1}=$ capacity in microfarads of electrometer, connexions, \&c., then the quantity of electricity which passes between the electrodes per second is equal to

$$
\frac{d_{1}}{\mathrm{~S}} \times \frac{1 \cdot 434}{1} \times \frac{c_{1}}{10^{6}} \times 3 \times 10^{9} \text { electrostatic units. }
$$


300 Mr. Mcllung on the Rate of Recombination of

But quantity of electricity = number of ions $x$ charge on an ion. $Q=$ number of ions, and let $\epsilon=$ charge on an ion :

$$
\begin{gathered}
\therefore \quad \frac{d_{1}}{\mathrm{~S}} \times \frac{1.434}{1} \times \frac{c_{1}}{10^{\circ}} \times 3 \times 10^{9}=\mathrm{Q} \times \epsilon . \\
\therefore \quad \mathrm{Q}=\frac{d_{1}}{\mathrm{~S}} \times \frac{1434}{1} \times \frac{c_{1}}{\epsilon} \times 3 .
\end{gathered}
$$

The value of $\mathrm{N}$ may be determined by the same formula if we substitute $d_{2}$ for the deflexion corresponding to $\mathrm{N}$ and $c_{q}$ for the capacity in the circuit. Substituting the menn value of the quantities experimentally observed, viz.:-

we obtain

$$
\begin{aligned}
& d_{1}=24 \cdot 0.7 \text { divisions of scale, } \\
& \mathrm{s}=93 \cdot 2 \quad, " \quad " \\
& c_{1}=.00380 \mathrm{~m} . \mathrm{f} .
\end{aligned}
$$

$$
\begin{aligned}
Q & =\frac{24 \cdot 07}{93 \cdot 2} \times \frac{1434}{1} \times \frac{.0038}{\epsilon} \times 3 \\
& =\frac{422}{\epsilon \times 10^{2}} .
\end{aligned}
$$

Also

$$
\begin{aligned}
d_{2} & =104.5 \text { divisions of scale, } \\
c_{2} & =\cdot 00016 \mathrm{m.f.} \\
\therefore \quad \mathrm{N} & =\frac{104.5}{93.2} \times \frac{1434}{1} \times \frac{.00016}{\epsilon} \times 3 \\
& =\frac{7722}{\epsilon \times 10^{4}} \\
\therefore \quad \frac{\mathrm{Q}}{\mathrm{N}^{2}} & =7.076 \times \epsilon ; \\
\therefore \quad a & =478.9 \times 7.076 \epsilon \\
& =3384 \epsilon .
\end{aligned}
$$

This value of $\alpha$ represents the menn value of a number of determinations.

If we substitute the value of $\epsilon$ obtained by Prof. J. J. Thomson, namely $6.5 \times 10^{-10}$, then $\alpha$ becomes $2.20 \times 10^{-6}$, which gives the absolute value of the coefficient.

This value of the coefficient of recombination agrees very closely with that obtained by Prof. Townsend for gases at atmospheric pressure (Phil. Trans. vol. exciii. 1899) by an 
entirely different method. The method which he used was that of sending a current of ionized air along a tube containing an insulated electrode connected to an electrometer. By taking the deflexion for the electrode at different positions along the tube, he was able to determine the rate at which the number of ions diminished in consequence of recombination. In that paper he gives the value as $3420 \epsilon$. The agreement between the two determinations is remarkably close considering the diversity of methods and apparatus employed by Prof. Townsend and myself.

The value of $\alpha$ which has been obtained by this determination for air at atmospheric pressure may be considered as the value of the coefficient of recombination for air at all other pressures, at least between the limits of 125 and 3 atmospheres, since it has been shown in a former part of this paper that $\alpha$ is independent of the pressure of the air.

It might be worth while to mention briefly the method which was employed to determine the electric capacities in the circuit, as it is rather an unusual one.

To determine the value of $\mathrm{N}$, the capacity in the circuit was simply that of the plate electrodes, the connexions, and electrometer. To determine $Q$, however, it was necessary to introduce a considerably larger capacity into the circuit, as the rate of movement of the electrometer-needle was too rapid without it. For this purpose, an air-condenser was connected in parallel, as shown at $\mathbf{M}$ in fig. 1 . It was necessary therefore to determine the capacity both of this condenser and of the electrometer, together with the connexions. To do this, the following method was used.

If the air between two insulated parallel plates be ionized by means of uranium oxide, and if one of the plates be charged while the other is connected to the electrometer; then the deflexion of the electrometer-needle per second is inversely proportional to the capacity in the circuit.

Two parallel zinc plates were therefore arranged horizontally in a closed metal box. The inclosing box was connected to earth, while the two plates were carefully insulated. On the lower plate a thin layer of uranium oxide was placed. The lower plate was connected to the battery, while the upper one was connected in parallel with the electrometer circuit. This of course introduced the additional capacity of the two parallel plates. The ratio of the capacity of these plates to that of the rest of the electrometer circuit was, however, determined by the method of mixtures.

The rate of leak was taken with a condenser of known capacity in the place of M, fig. 1 . Then the known capacity 
was removed and the air condenser, to be determined, introduced, and the rate of leak taken again. A third observation was then taken without any condenser in the circuit at all.

Now by comparing the last observation with that obtained when the condenser of known capacity was in circuit, the capacity of the electrometer, connexions, and plate-electrodes was determined. Then, having obtained this capacity, that of the air condenser was determined by a comparison of the deflexions obtained in the second and third observations.

This method of determining capacities was found to be much more satisfactory than the method of mixtures. The capacity of the electrometer, connexions, \&c., was found to be -00016 of a microfarad, while that of the air condenser was .00364 of a microfarad.

\section{Consideration of other Gases.}

This question of recombination of ions was investigated also in the case of carbonic acid gas and hydrogen gas. The same method was employed as for air. The recombination of the ions in these gases under difterent pressures could not, however, be satisfactorily investigated, as the supply of gas was limited in each case and no means of filling the cylinder with these gases, under increased pressure, was at our disposal. The results given here, therefore, for carbon dioxide and hydrogen are for these gases under a pressure just slightly above atmospheric pressure. The pressure was a few millimetres above that of the atmosphere, in consequence of the pressure of a short column of the acid, from which the gas was generated in each case, in the generating apparatus. In the case of both hydrogen and carbon dioxide, it was tested whether the ions in these gases followed the same law of recombination as was found to hold in the case of air. The law was found to be the same in these gases as in air. A comparison of the experimental and theoretical curves is shown for carbon dioxide and hydrogen in fig. 8.

The agreement between theory and experiment in these two gases is therefore seen to be as close as is the case with air. The indications are then that this law of recombination of the ions is an invariable one. It has been shown conclusively by this research, that the rate at which ions recombine is governed by this law in the cases of air, carbon dioxide, and hydrogen. 
Ions in Gase: under Different Pressures.

Fig. 8.

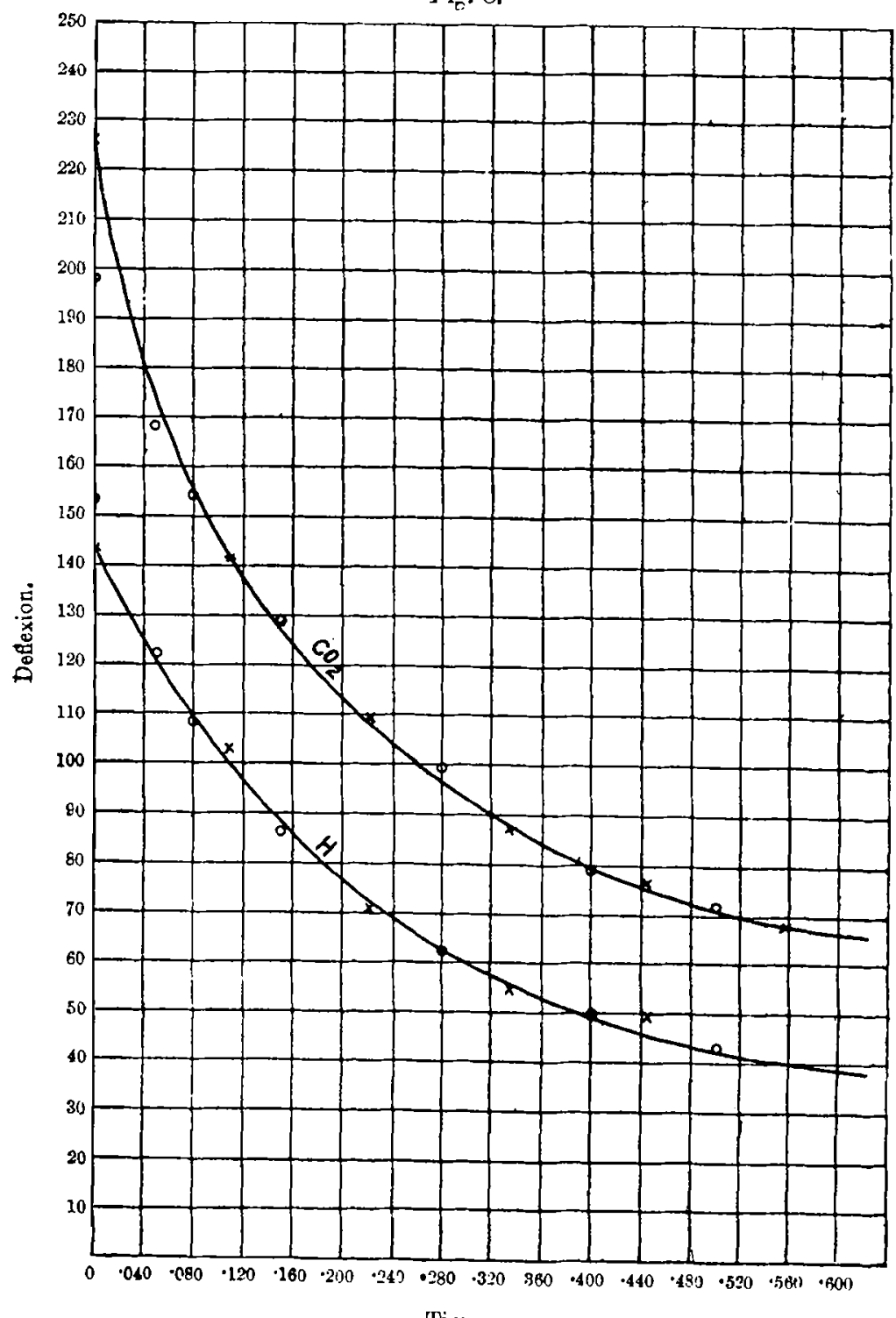

Time. 
The absolute value of the coefficient $a$ for these two gases was also determined. Carbon dioxide presented a little difficulty, as the determinations made on it did not keep perfectly constant from day to day. A considerable number of determinations were made, however, extending over several days and the mean of these values taken. This value of $\alpha$ was found to be $3492 \times \epsilon$, which on substituting the value of $e$ becomes $2.27 \times 10^{-6}$. This is practically the same as the value of the coefficient obtained for air.

The corresponding determination for hydrogen resulted in a value only about 87 per cent. of the value for air. The actual result for hydrogen was $2938 \times \epsilon$, which becomes $1.91 \times 10^{-8}$ on substituting the value of $\epsilon$. From this it appears that, although the coefficient of recombination is a constant for any gas, it has not necessarily the same value for all gases.

Referring again to Prof. Townsend's paper (Phil. Trans. Roy. Soc. vol. cxciii. 1899) we find that these results are again in close agreement with his. The result for hydrogen given above is also the mean result of several determinations.

\section{Removal of Nuclei.}

'Throughout this investigation, care was taken to thoroughly dry the gas and to remove dust particles from it before allowing it to pass into the cylinder in which the measurements were made on it. For this purpose, the air which was used in the cylinder was first passed slowly through an iron tube (about two feet in length) filled with calcium chloride, to remove the moisture, and then through a bulb containing cotton-wool, to remove any dust particles.

When hydrogen and carbon dioxide were used, the gas was passed through a series of drying-bottles containing small pieces of pumice-stone moistened with strong sulphuric acid, and thence through the cotton-wool as before. These precautions were taken of course on account of the fact, which has been previously investigated ( $\mathrm{E}$. Rutherford, Phil. Mag. Nov. 1897), that when any nuclei are present in the gas, the ions collect about these nuclei and become neutralized. The number of ions decreases therefore much more rapidly when any nuclei are present in the gas than it does if the gas is quite free from these particles. As the object was to determine the rate of decrease in the number of ions due simply to the recombination with one another, the precantion was therefore taken to remove all foreign particles from the gas as far as possible. 
Summary of Results.

The results obtained from this research may be summarized as follows :-

(1) The rate at which the ions recombine in ionized air is determined by the same law, namely, $\frac{d n}{d t}=-\alpha n^{2}$, no matter what the pressure of the gas may be, at least between the limits of 125 of an atmosphere and 3 atmospheres.

(2) The coefficient of recombination for the ions in air is independent of the pressure of the air for the same range of pressures as above.

(3) The absolute value of this coefficient has been determined and has heen found to be $3384 \mathrm{e}$, or on substituting the value of the charge on an ion it becomes $2 \cdot 20 \times 10^{-6}$.

(4) Hydrogen and carbon dioxide were also examined, and the same law of recombination was found to hold true as for air.

(5) On determining the coefficient for carbon dioxide, it proved to be practically the same as for air, while the coefficient for hydrogen is only about 87 per cent. of the ralue for air.

In conclusion, 1 wish to express $m y$ sincerest thanks to Prof. Rutherford, at whose suggestion this research was undertaken, and who has so kindly rendered me the most valuable assistance by his advice and suggestions throughout the whole investigation.

Macdonald Physical Laboratory,

McGill College, Montreal, July 4,1901 .

XXXI. Condensation of the Vapours of Organic Liquids in Presence of Dust-free Air. By F. G. Donnan, M.A., Ph.D.*

[Plate V.]

\section{$\S 1$. Introduction.}

THE question why some liquids act as ionizing solvents while others do not, is one of fundamental importance in the theory of electrolytic dissociation, yet, although various suggestions have been made, no satisfactory explanation of these differences has hitherto been given. The experiments

* Communicated by the Author.

Phil. Mag. S. 6. Vol. 3. No. 15. March 1902. 